\title{
Robust Positioning Performance in Indoor Environments
}

\author{
Allison Kealy ${ }^{1}$, Guenther Retscher ${ }^{2, *}$, Yan $\mathrm{Li}^{3}$, Thomas Gonzales ${ }^{4}$, Salil Goel ${ }^{5}$, Jelena Gabela ${ }^{3}$ \\ ${ }^{1}$ School of Science, RMIT University, City Campus, Melbourne, Australia \\ ${ }^{2}$ Department of Geodesy and Geoinformation, TU Wien, Gusshausstrasse 27-29, 1040 Vienna, Austria \\ ${ }^{3}$ Department of Electrical and Electronic Engineering, The University of Melbourne, Melbourne, Australia \\ ${ }^{4}$ French Civil Aviation University, Toulouse, France \\ ${ }^{5}$ Department of Civil Engineering, Indian Institute of Technology Kanpur, Uttar Pradesh, India
}

\begin{abstract}
Increasingly, safety and liability critical applications require GNSS-like positioning metrics in environments where GNSS cannot work. Indoor navigation for the vision impaired and other mobility restricted individuals, emergency responders and asset tracking in buildings demand levels of positioning accuracy and integrity that cannot be satisfied by current indoor positioning technologies and techniques. This paper presents the challenges facing positioning technologies for indoor positioning and presents innovative algorithms and approaches that aim to enhance performance in these difficult environments. The overall aim is to achieve GNSS-like performance in terms of autonomous, global, infrastructure free, portable and cost efficient. Preliminary results from a real-world experimental campaign conducted as part of the joint FIG Working Group 5.5 and IAG Sub-commission 4.1 on multi-sensor systems, demonstrate performance improvements based on differential Wi-Fi (DWi-Fi) and cooperative positioning techniques. The techniques, experimental schema and initial results will be fully documented in this paper.
\end{abstract}

\section{Introduction}

This paper reports about an experiment, conducted in a GNSS-denied/challenged, indoor environment at the Queen Victoria Market (QVM) located in Melbourne, Australia. The overall aim of this campaign was to assess the feasibility of achieving GNSS-like performance for ubiquitous positioning at affordable cost efficiency. In the experimental campaign, we developed a cooperative system comprising of seven pedestrian users equipped with smartphones using an integration of signals such as Wi-Fi (Wireless Fidelity) and UWB (Ultra-Wide Band) with the objective of achieving precise positioning in indoor environments. In this paper, the set-up of the experiments and their characteristics are presented, and selected results are discussed.

For Wi-Fi Received Signal Strength (RSS) based indoor positioning, fingerprinting is one of the widelyemployed methods which can offer relatively high positioning accuracy. Conventional fingerprinting methods, however, normally perform a training using a site survey only once in the beginning and thereafter only if the environment changes significantly, which are not resistant to dynamic environment changes. Continuously updating the fingerprint database is a new way to enhance the achievable positioning accuracy. For that purpose, a Differential Wi-Fi (DWi-Fi) scheme by analogy to DGNSS is applied. It is a network calibration method based on reference stations realized by low-cost Raspberry Pi units which is able to derive the correction parameters in real-time. In this way, the measured RSS values at the user's side are corrected, the fingerprinting database is continuously updated, and thus an adaption to the possible changes in the dynamics of the environment is achieved.

Furthermore, in this study, the derivation and integration of UWB ranges for positioning of mobile users is investigated. These UWB ranges serve either as more precise reference for the Wi-Fi derived ranges or are integrated into the overall positioning solution. Due to a collocation of the UWB transmitters forming the anchors of the UWB positioning system with the Raspberry Pi units for Wi-Fi positioning in the test site an integration of the two system solutions is possible.

The paper is organized as follows: Firstly, key requirements and performance parameters for PNT (Position, Navigation and Timing) are identified in section 2. Section 3 presents Wi-Fi positioning based on the differential approach (termed Differential Wi-Fi, DWi-Fi) followed by the UWB positioning scheme in section 4 . Then the test area is introduced in section 5 and the main results in section 6 . Section 7 presents concepts for Wi-Fi signal distribution interpolation approaches realized by so-called radio maps -and their use in the DWi-Fi scheme. Concluding remarks and an outlook are drawn in the final section 8 .

\footnotetext{
* Corresponding author: guenther.retscher@tuwien.ac.at
} 


\section{Key Requirements and Performance Parameters}

As stated in the 2017 GNSS market report [1] the key GNSS requirements and performance parameters are summarized in the following. It must be noted that they are also applicable for any other PNT applications not involving only GNSS but also other sensors and technologies which are additionally and independently used. The key parameters are:

- Availability: percentage of time over a specified time interval that a sufficient number of satellites are transmitting a usable ranging signal within view of the user. Values vary greatly according to the specific application and services used, but typically range from 95-99.9\%.

- Accuracy: the difference between true and computed position (absolute positioning). This is expressed as the value within which a specified proportion of samples would fail if measured. Typical values for accuracy range from tens of meters to centimeters for 95\% samples. Accuracy is typically stated as 2D (horizontal), 3D (horizontal and height) or time.

- Continuity: ability to provide the required performance during an operation without interruption once the operation has started. Continuity is usually expressed as the risk of discontinuity and depends entirely on the timeframe of the application (e.g. application that requires 10 minutes of uninterrupted service has a different continuity figure than one requiring two hours of uninterrupted service, even if using the same receiver and services). A typical value is $1 \times 10^{-4}$ over the course of the procedure where the system is in use.

- Integrity: the measure of trust that can be placed in the correctness of the position or time estimate provided by the receiver. This is usually expressed as the probability of a user being exposed to an error larger than alert limits without warning.

- Time To first Fix (TTFF): a measure of a receiver's performance covering the time between activation and output of position within the required accuracy bounds. Activation means subtly different things depending on the status of the data the receiver has access to.

- Robustness: the ability of systems or system elements to withstand a level of interference and/or jamming without significant degradation or loss of performance.

- Authentication: the ability of the system to assure that they are utilising signals and/or data from trustworthy source (e.g. GNSS constellation), and thus protecting sensitive applications from spoofing threats.

If these key requirements and performance parameters are applied, for instance, for Wi-Fi or UWB positioning a similar statement or meaning can be formulated. Regarding availability the number of transmitters (UWB stationary transmitters or Wi-Fi Access Points) replaces the number of satellites. Especially integrity is often neglected and not paid full attention. It can be seen as a very important key parameter. The way that integrity is ensured and assessed, and the means of delivering integrity related information to the user are highly application dependent. TTFF in the case of Wi-Fi positioning is highly correlated to the received signal strength (RSS) scan duration of a certain mobile device. This is especially important in kinematic positioning. As seen in [14] the appearing scan durations can vary significantly for different smartphones which results in a different level of achievable positioning accuracy in dependence of the walking speed in the case of pedestrian navigation. For different users robustness may have a different meaning, such as the ability of the solution to respond following a server shadowing event. Here, robustness is defined as the ability of the solution to mitigate interference.

Other requirements and performance parameters are power consumption, resiliency, connectivity, interoperability and traceability [1]. Especially in the case of mobile devices power consumption is still very critical to provide a long-term solution possibility. Resiliency is the ability to prepare for and adapt to changing conditions, such as it is the case for Wi-Fi RSS signal variations and fluctuations. To encounter for their influence a new differential scheme is developed which is briefly discussed in the following section 3 .

\section{Differential Wi-Fi (DWi-Fi) Scheme}

In all common RSS-based Wi-Fi positioning approaches scanned absolute RSS values are used either directly in fingerprinting or for the RSS to range conversion using path loss models for trilateration. In a differential approach referred to as Differential Wi-Fi (DWi-Fi) trilateration - by analogy to the commonly employed Differential GNSS - continuous RSS measurements performed at reference stations (RSs) distributed in the area of interest are used to derive real-time corrections for the mobile users. Instead of theoretical path loss models this approach utilizes continuous RSS scans carried out during user localization to improve the positioning accuracies, its solution integrity and reliability [13, 17]. For a low-cost realization Raspberry Pi units serve as reference stations (RSs) and Access Points (APs) at the same time scanning and emitting Wi-Fi signals. Using these devices the RSS scans are recorded together with their MAC addresses of the Wi-Fi APs continuously. Furthermore, relative RSS values instead of absolute numbers either between consecutive epochs or different APs are used in addition. If different APs can be received at a certain location the RSS values can be ordered in dependence of their RSS of the AP. At a second location in the area of interest the order is usually different. Then the difference in order sequence of the AP RSS values between the different locations can be used to match the current RSS measurements to the correct location. Consider, for example, a descending order of RSS where AP1 has higher values than the other APs. On a different location this order might be reverse. If the difference is 
significant then it is easier to determine where the smartphone is currently located. The difference in order results most likely from physical differences in building structures as in one room the situation is completely different than in another room. Furthermore, different consecutive epochs of RSS measurements can be considered in assignment of the correct location. Due to the significant temporal variation of RSS it is then easier to match the users' location to the correct one. If one considers long-time measurements of RSS at reference stations then in real-time the change of RSS of all visible APs can be determined. This approach results into a fusion of different localization techniques as RSS observations are performed continuously on the aforementioned RSs and used to derive dynamically changing maps of RSS distributions, the so-called radio maps, in real-time. Thus, the principle is the fusion of the location fingerprinting and trilateration techniques combining the advantages of both methods [12]. The main advantage is that no lengthy training phase requiring high workloads and frequent re-calibration as in standard fingerprinting is required [16].

\section{Ultra-wide Band (UWB)}

In addition to low-cost Wi-Fi technology, in this study an UWB (Ultra-wide Band) system was employed. Due to high-bandwidth communication characteristics and the high standard clock synchronization between transmitter and receiver, UWB radio technology offers strong multipath resistance capabilities and to a certain extend penetrability for building materials leading to high accuracy range measurements compared to other radiobased technologies [11]. Commercially available UWBs rely on two way TOF (time-of-flight) to realize the distance between the UWBs. This study employed the Time Domain's PulsON ${ }^{\circ}$ OEM [2] modules that uses the coherent transmission of sequences of short duration, low duty cycle radio frequency pulses to provide high quality ranging measurements. Fig. 1 shows the Time Domain's PulsON® OEM transmitter set-up on a tripod.

\section{Test Site Characteristics, Sensor Deployment and Smartphone Specifications}

Fig. 2 shows impressions from the test site in a part of Queen Victoria Market (QVM) in Melbourne, Australia, indicating the sensor deployment and reference points RPs along the chosen circular trajectory. Seven smartphone users were walking along this closed-loop trajectory around the shops comprising of four corridors with 16 RPs distributed in a test grid of $4 \mathrm{~m}$. Seven Raspberry Pi units were deployed to serve both as APs or
RSs at the same time and six static UWBs. They were surveyed by a total station for ground truth. Both kinematic and stop-and-go measurements were carried out [15]. In the first case, a continuous recording of the RSS scans was performed and in the second case up to ten RSS scans on each of the 16 RPs were carried out. The users started at different RPs while walking behind each other along the trajectory. Moreover, the inertial sensor observations from the smartphones were recorded as well. Table 1 shows the employed smartphones specifications and their start reference points along the trajectory shown in Fig. 2.

The role of UWB system (see Fig. 1) was, firstly, to provide a high quality trajectory to serve as a ground truth for the DWi-Fi solution, and secondly to provide further location information (e.g. in the form of control points along a trajectory) for improving the DWi-Fi positioning solution. In this paper, the UWB measurements are used only to produce the range measurements between the moving user and stationary UWB nodes, as a means of quality assurance of the mobile user trajectory. A further step is a comparison with the Wi-Fi derived ranges. This is facilitated as the UWB anchors fixed on the walls were collocated with the Raspberry $\mathrm{Pi}$ units for Wi-Fi positioning.

Table 1. Smartphone user configuration and start reference points.

\begin{tabular}{|l|l|c|}
\hline User ID & Smartphone & $\begin{array}{c}\text { Start reference } \\
\text { point }\end{array}$ \\
\hline 1 & Sony Xperia Z1 & 1 \\
\hline 2 & Samsung Galaxy S3 & 3 \\
\hline 3 & Samsung Galaxy S3 & 5 \\
\hline 4 & Motorola G3 - MotoG3 & 9 \\
\hline 5 & Huawei Mate 7 & 11 \\
\hline 6 & Huawei Honor 8 & 13 \\
\hline 7 & $\begin{array}{l}\text { Samsung Galaxy GT- } \\
\text { S7262 }\end{array}$ & 15 \\
\hline
\end{tabular}

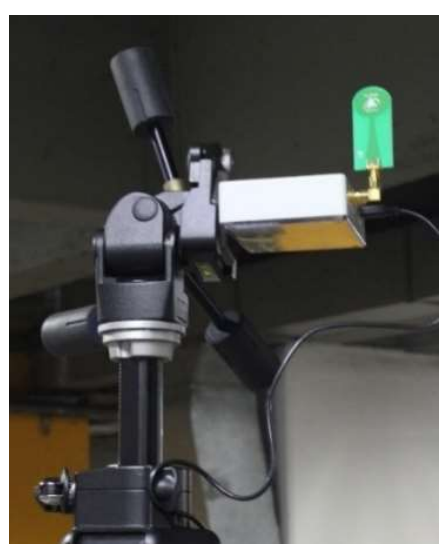

Fig. 1. Time Domain's PulsON® OEM. 

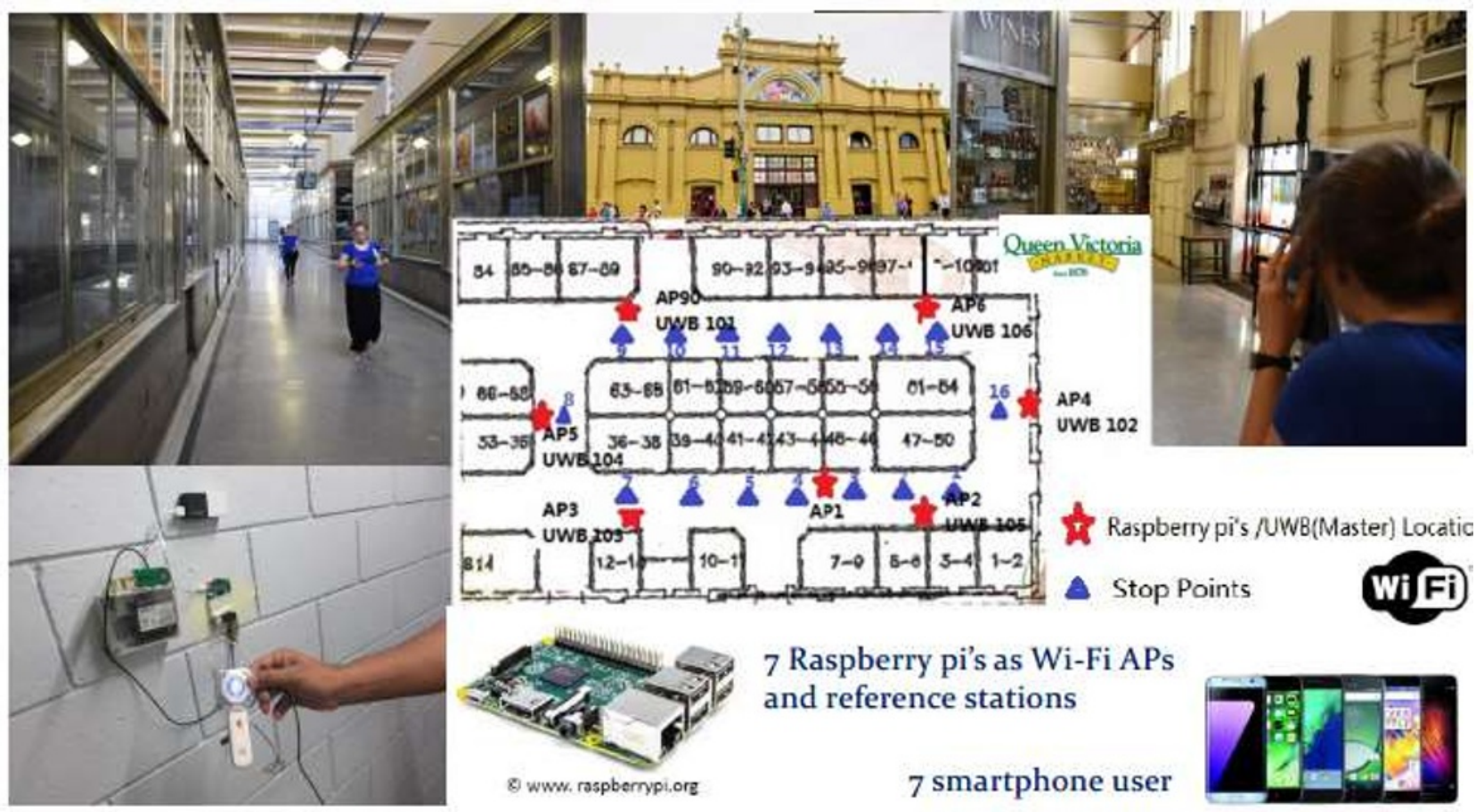

7 Raspberry pi's as Wi-Fi APs and reference stations

7 smartphone user

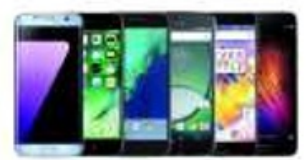

Fig. 2. Impressions from the test site in QVM showing Raspberry Pi and UWB sensor deployment and 16 reference stop points.

\section{Analyses and Main Results}

In outdoor environments, the positioning solution is derived as usually primarily from GNSS and relative range observations among pedestrians. In indoor and transition environments, the localization solution is estimated using relative range observations among pedestrians and Wi-Fi RSS measurements. In this section, selected results of the measurement campaign in QVM are presented.

\subsection{Wi-Fi Positioning Results}

Wi-Fi is nowadays kind of ubiquitous in our environments. This pervasiveness of Wi-Fi signals allows to use parameters like RSS to estimate the distance between mobile devices from which Wi-Fi signals are exchanged. In the conducted work, Raspberry Pi units (RPis) served as Wi-Fi Access Points. Using path loss models the relationship between the measured RSS and the range is derived [17] and knowing the coordinates of the respective Access Points then the position of a user can be estimated.

In the first stage of the analyses, the following properties of the RSS measurements at the Raspberry Pi units were investigated [7]:

- Mean, variance and standard deviation of the signal quality,

- Mean, variance and standard deviation of the RSS measurements, and
- The ascending ranking order of those two parameters, more particularly for RSS measurements.

The aim of checking their mean and standard deviation is to determine for each Raspberry Pi unit serving as Access Point (AP) which is the best one with the best quality and/or highest RSS. Moreover, it is interesting to know their ascending ranking order in terms of power (like in $\mathrm{dBm}$ or Watt for the RSS) for each time of the experiment. This gives an idea of the architecture built by the Raspberry Pi units. In a matter of fact, a high RSS means a shorter distance between the RPi units (according to path loss models). Then, a weak RSS means a longer distance between two APs. And if one RSS close to a RPi is weaker than it should be, this may indicate a connection loss, something that for instance AP2 underwent during the experiment. Mean and variance are affected by extreme values. That's why also the percentage of time of each RSS ranking order was investigated. In other words, the percentage of time where a RSS from one $\mathrm{AP}_{\mathrm{i}}$ is higher than a RSS from a second $\mathrm{AP}_{\mathrm{j}}$ was investigated.

From all the measured RSS data unfortunately not all Access Points recorded at once. So among those seven units, only four could be used to perform lateration, which are AP2, AP4, AP5 and AP6. For 2D lateration four APs provide at least some redundancy.

Fig. 3 shows a visualization of the RSS value distributions. The two horizontal axes in the diagrams show either the AP number or the reference points RPs with their Point ID. The absolute measured RSS values from up to 10 scans at each RP are shown on the vertical axis [16]. As it can be seen from the plots, the absolute RSS values of the seven APs vary significantly in 
dependence on the respective location of the user. Such a situation is favourable for positioning if the absolute RSS values are used since the match to the current location can be achieved more easily. Furthermore, a quite similar distribution for the different APs can be seen when comparing the different smartphones. The absolute RSS values, however, can differ in dependence of the smartphone.

In the following, the ascending ranking order of the Raspberry units was investigated. As can be seen from the RSS value sequences in the top Fig. 4 for AP2 in respective to the other three APs a clear order is visible. Thereby, the RSS relationship between AP2 and AP4 is depicted in blue, with AP5 in red and with AP6 in green, respectively. The ascending ranking order is very stable. The RSS order AP5 $<$ AP6 $<$ AP4 occurs more than 98\% of the time. The reason for this is that AP5 is further away from AP2 than AP6. And that's the case as can be seen in the map of the test site in QVM shown in Fig. 2. This is also the case for AP4. In fact, if a stable RSS ranking order occurs, the position of the electronic devices can be estimated with a good precision.

A further result was that a connection loss between the Raspberry Pi units happened less than $1 \%$ of the whole time, meaning that the APs performance was excellent, almost perfect.

The result of this investigation for AP6 is shown in the lower Fig. 4. The RSS relationship between AP6 and AP2 is depicted in black, with AP4 in yellow and with AP6 in red. Here, the RSS ranking order is completely different as before as APs which are further away from AP6 do not result always in lower RSS values. Furthermore, the noise of the RSS numbers is higher.

Thus, the consistency of the ranking order was further investigated more in detail. It is defined as follows: equality between the RSS $A_{i}=>A P_{j}$ and RSS $\mathrm{AP}_{\mathrm{j}}=>\mathrm{AP}_{\mathrm{i}}$. Equal consistency means in other words that if a signal coming from $\mathrm{AP}_{\mathrm{i}}$ to $\mathrm{AP}_{\mathrm{j}}$ is the same in reverse from $A P_{j}$ to $A P_{i}$. It was also verified if the RSSs were distributed according to a Gaussian law to check some properties on them like computing trust intervals. Fig. 5 shows as an example the consistency of the RSS values sequence between AP2 and AP4. Consistency could never been verified in the whole data set. There is always a significant difference between the RSS $\mathrm{AP}_{\mathrm{i}}=>\mathrm{AP}_{\mathrm{j}}$ and RSS $A P_{j}=>A P_{i}$. In the case shown in Fig. 5 for the consistency between AP2 and AP4 a relatively constant difference of around $11 \mathrm{dBm}$ occurred which can be seen as a significant bias. This bias was stable over the whole common time interval. In general, this bias occured in all AP combinations with a value of around $10 \mathrm{dBm}$ on average.

To see if on the RPi data can be relied on the RSS correlation was investigated. It was tested if the signals are independent between each others or if there is a form of correlation. From the correlation matrix it was found that the correlation factors were very weak of all possible $\mathrm{RPi}$ couples. Thus, the hypothesis validated the independency of the signals coming from the Access Points. Table 2 is an example of the correlation matrix with AP5 as a receiver (so correlation tested here will be between AP2-AP4, AP2-AP6 and AP4-AP6).

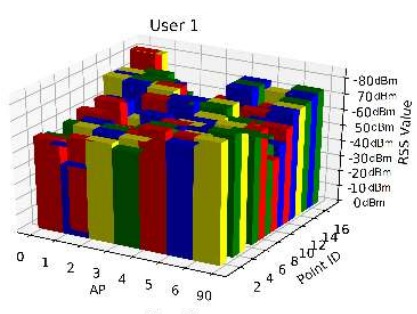

User 3

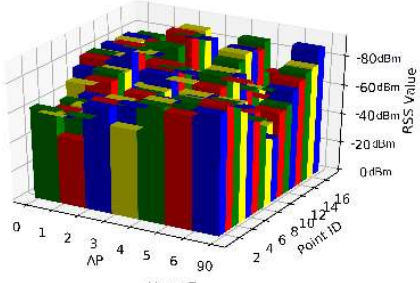

User 5
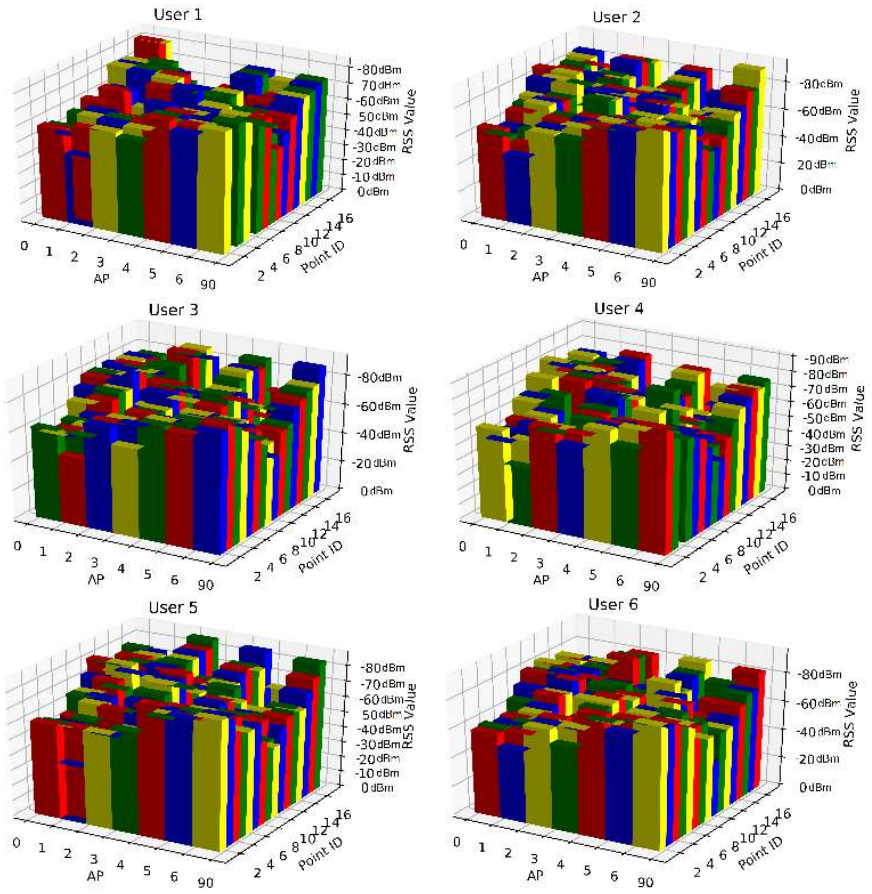

User 4

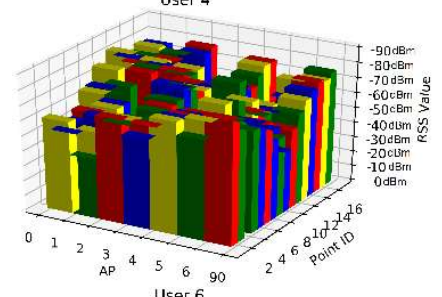

User 6

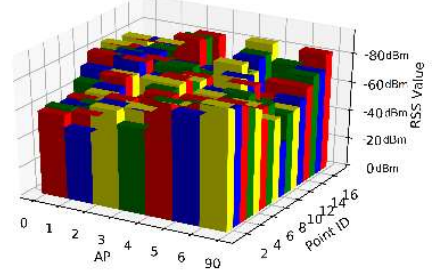

User 7

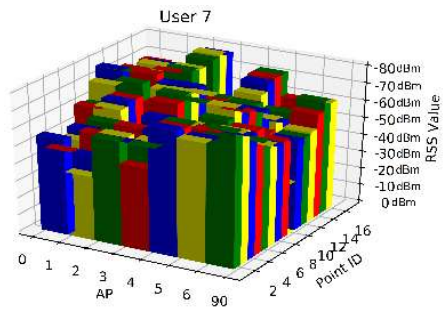

Fig. 3. Comparison of the absolute RSS values on the 16 RPs collected by seven different smartphone users (Source: [16]).

Table 2. RSS correlation matrix with AP5 recorded data, showing correlation between different signals coming to AP5

\begin{tabular}{|c||c|c|c|}
\hline & AP2 & AP4 & AP6 \\
\hline \hline AP2 & 1 & -0.0267 & -0.708 \\
\hline AP4 & -0.0267 & 1 & -0.0103 \\
\hline AP6 & -0.0708 & -0.0103 & 1 \\
\hline
\end{tabular}

\subsection{UWB Positioning Results}

Pedestrians rely on relative UWB ranges (including ranges between pedestrians, and ranges between pedestrian and UWB anchors) and Wi-Fi measurements, for localizing all users cooperatively. Fig. 6 shows a plot of range measurements as observed by two pedestrians with respect to the UWBs as a function of time. Thereby pedestrian user 1 started at point 9 and the second user at point 1 . The measurements were performed in stop-andgo manner. The two highlighted time interval windows in Fig. 6 can be seen more clearly in detail in Fig. 7 (zoomed view 1 and 2). The region where the UWB ranges are constant are the regions where the pedestrians were standing for a certain period of time. It is seen that a maximum range of $20 \mathrm{~m}$ was achievable in the QVM indoor environment. Significant communication drops between the pedestrians and RPi as seen in Fig. 6. For example, no communication of the pedestrian with the 

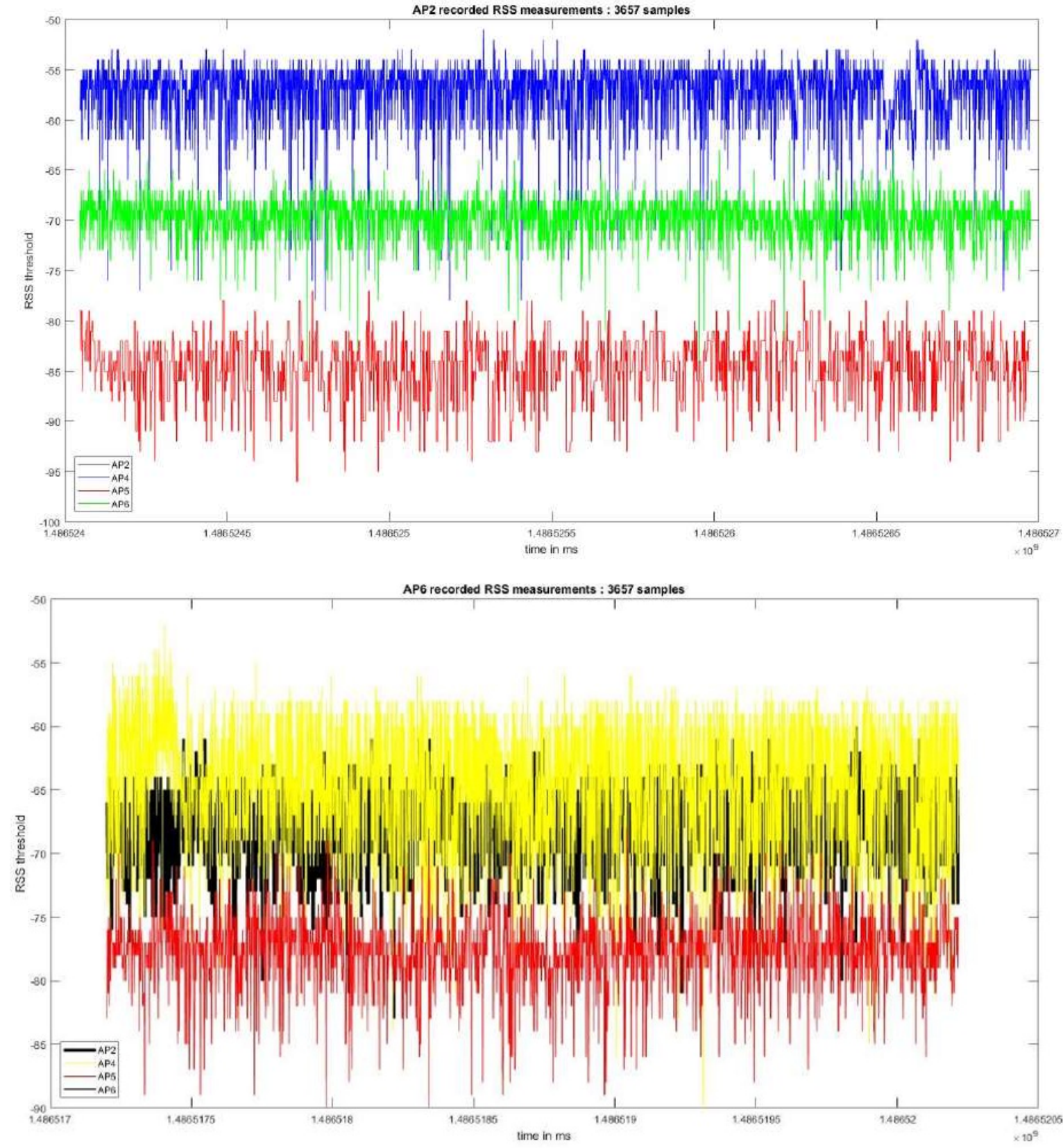

Fig. 4. RSS values sequence of AP2 (top) and AP6 (bottom) seen from the other APs.

$\mathrm{RPi}$ is observed between time instants $2.6 \times 10^{4} \mathrm{~s}$ to $2.7 \times 10^{4} \mathrm{~s}$ and from time instant $2.8 \times 10^{4} \mathrm{~s}$ to $3.1 \times 10^{4} \mathrm{~s}$. This is most likely due to the complex nature of the indoor environment and non-availability of direct Line of Sight (LOS) between the pedestrian and RPi. It is obvious that non-availability of communication signals to the pedestrians will affect their indoor localization performance. One of the ways to improve communication capabilities in indoor environments is to deploy more number of reference RPi UWBs. Several interesting observations regarding the behaviour of UWBs in indoor environments can be made from Figures 6 and 7. It is observed that the points occupied by the pedestrians can be easily identified in the plots shown in Fig. 7. This is represented by the regions where the UWB range measurements are relatively constant (since the data is collected in a stop-and-go manner). Even when the pedestrian is stationary at a given location, significant sudden changes in the range observations can be observed. For example, at time instants $2.62 \times 10^{4} \mathrm{~s}$ and $2.8 \times 10^{4} \mathrm{~s}$, sudden jumps in the range observations are visible. This is most likely because of multipath in indoor environment in the UWB observations [5]. Similar corruption by the outliers in the ranges derived from UWBs in complex environments has been previously reported by authors in $[4,5]$. Such outliers should be accounted for, within the cooperative state estimation framework. One such framework for incorporating corrupted UWB range measurements in a cooperative estimation framework is presented by authors in [5]. Further, it is interesting to observe that the nature of the UWB signals observed by both the pedestrians are very similar. For example, the UWB signals as observed by pedestrian 1 in two different corridors is seen (in Fig. 7) 


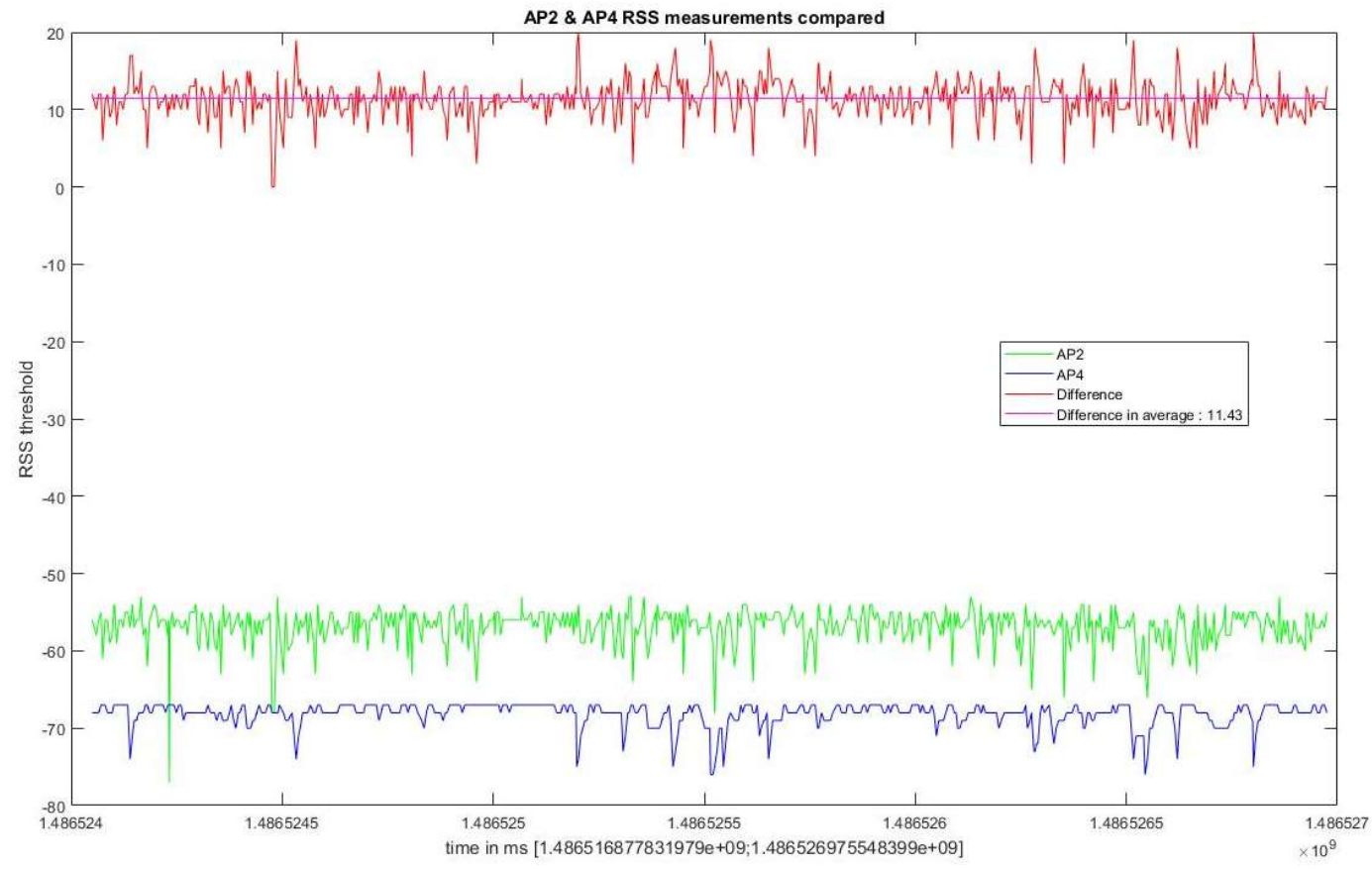

Fig. 5. Consistency of the RSS values sequence between AP2 and AP4.

between time instants $2.56 \times 10^{4} \mathrm{~s}$ to $2.58 \times 10^{4} \mathrm{~s}$ and $2.74 \times 10^{4} \mathrm{~s}$ to $2.76 \times 10^{4} \mathrm{~s}$. Similarly, the UWB signals observed by pedestrian 2 in the same two corridors are shown between time instant $2.6 \times 10^{4} \mathrm{~s}$ to $2.63 \times 10^{4} \mathrm{~s}$ and $2.78 \times 10^{4} \mathrm{~s}$ to $2.81 \times 10^{4} \mathrm{~s}$ in Fig. 7. A careful analysis of the UWB observations between these instants reveals that the pattern of the UWB range variation as observed by two pedestrians is very similar. This is to be expected since the nature of UWB range variation is dependent on the pedestrian movement (given the location of the RPi) and one pedestrian is right behind the second pedestrian, and thus, following the same trajectory.

The position estimates of both the pedestrians can be known if at least three range observations from reference RPi UWBs are available to each pedestrian [6]. However, given the limited number of available UWB sensors, achieving at least three range observations in indoor environments for extended periods is found to be rather challenging. In the experiments described in this paper, it is found that three or more range observations are observed rarely by the pedestrians, while 1-2 UWB observations were available most of the time during the experiment. This is due to the complex indoor environment and the insufficient number of UWBs deployed during the experiment. It is because of these reasons that pedestrian locations could not be derived from UWB observations only. Some of the key observations of these experiments is that a large number of UWBs need to be deployed depending on the complexity of the indoor environment to ensure availability of at least three range observations, and that ranges derived from UWBs are corrupted by outliers, which should be accounted for, within the cooperative state estimation framework.

\section{Wi-Fi Radio Map Interpolation and DWi-Fi}

In analyses of the Wi-Fi measurements the next step is the creation of maps of RSS distribution for the whole test site. These maps are reffered to as radio maps. In our study, the concept of radio map estimation is based on DWi-Fi, so using two data files, one for RSS $A_{i}=>A P_{j}$ and one for RSS $A P_{j}=>A P_{i}$ to determine one distance. The method of Voronoï Tessellation, based on geometrical theory, allows to conduct the estimation of all APs. For that purpose an interpolation method has to be applied to estimate the RSS for each point on the map. For this interpolation several methods exist, but it is necessary to achieve a good balance between the accuracy of the algorithm and its speed or efficiency. Thus, mainly two options shall be applied, i.e.:

- Linear interpolation: it is quite simple, but not so accurate. In fact, as the electromagnetic signals undergo an exponential decrease in distance the term of power evolves according to a logarithmic function. So opting for a linear interpolation is not always suitable.

- Delaunay algorithm based on Voronoï cells. Note that Delaunay and Voronoï concepts are linked because the the Delaunay triangulation is the dual problem of Voronoi diagram. 


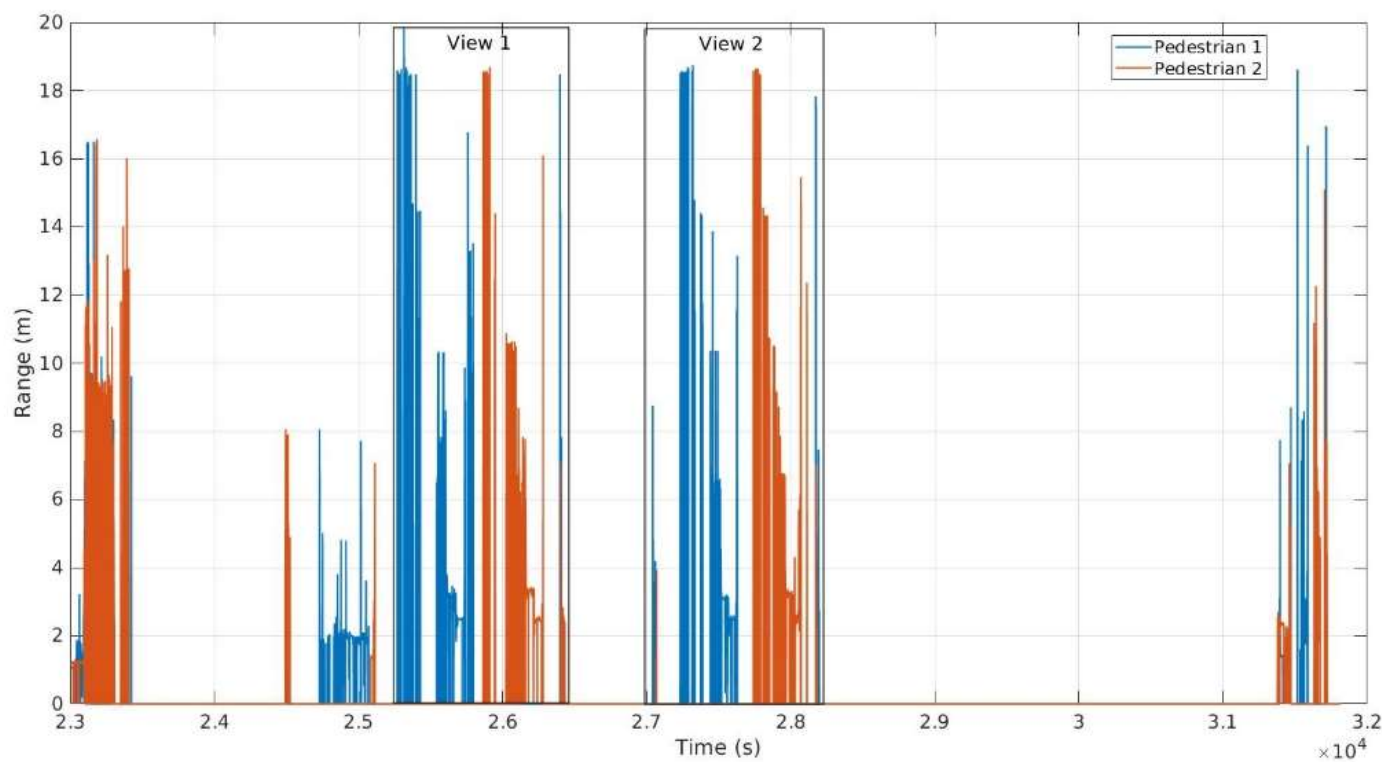

Fig. 6. Plot of range observations from the UWBs for two pedestrian users over time.
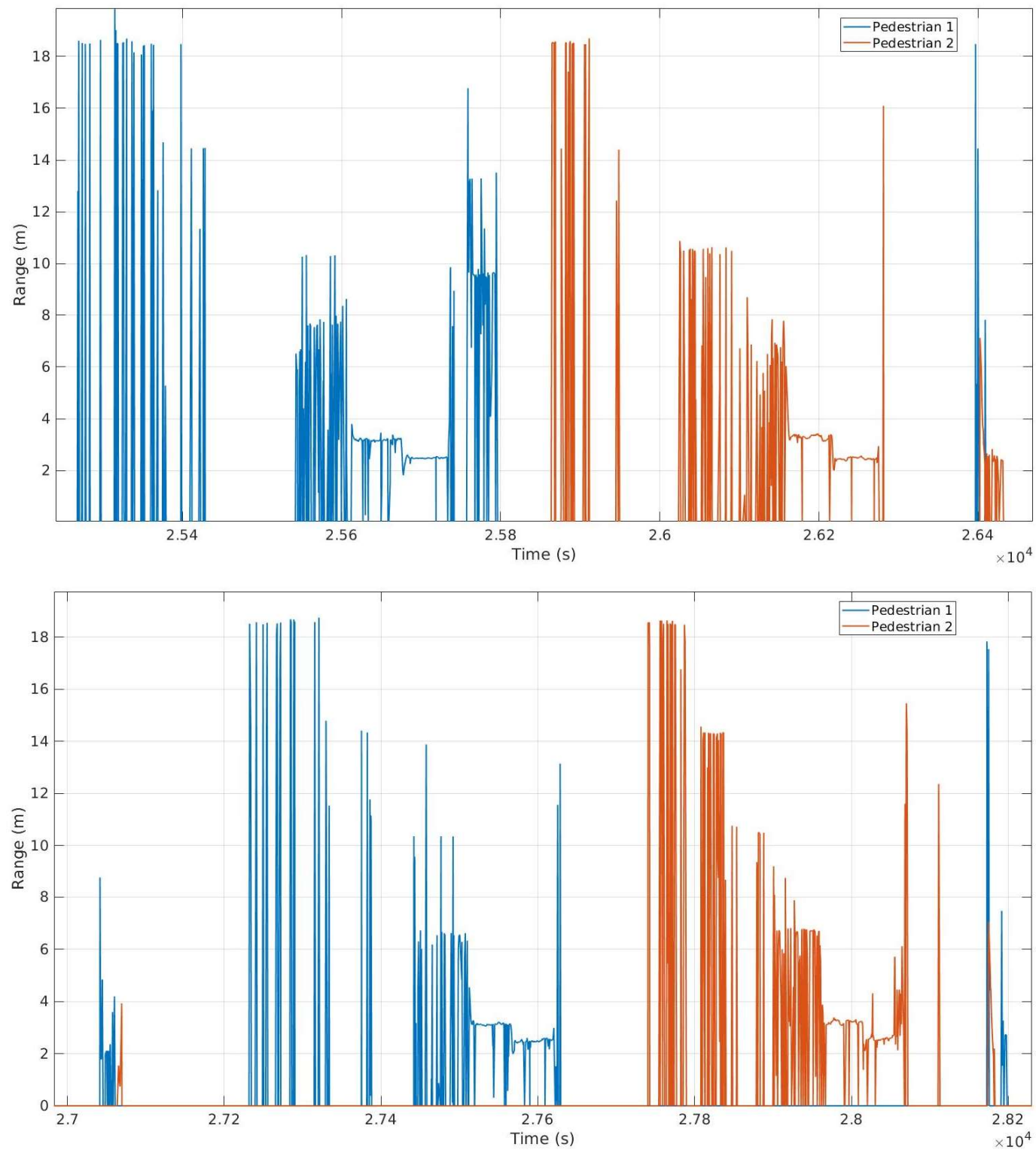

Fig. 7. Zoomed views 1 and 2 of the UWB range observations for the two pedestrians. 
The principle of the second interpolation approach is that a Voronoï diagram consists of so-called from Voronoï cells which are are defined by segments drawn between two points. Starting from a cloud of points, only some points are going to be linked between each others $[3,10]$. More precisely, the Voronoï/Delaunay method uses the nearest neighbour interpolation [9] giving a smooth approximation of the interpolated function. More accurate than linear interpolation, the building of the Voronoï tessellation brings advantage in the following two steps: Firstly, to quickly estimate the localization of all the Access Points, and secondly, to estimate signal fading parameters. To sum up it can be said that the Voronoï/Delaunay algorithm provides the possibility to interpolate the RSS function. In other words, it is going to perform a smooth interpolation of the function $f(x, y)=$ RSS where $(x, y)$ represents the position coordinates of a point $P$ located in the map (in 2D) and RSS the value of the RSS in $\mathrm{dBm}$ for this point. But above all, a function is needed to interpolate, or better said a base which could be used to estimate the RSS everywhere on the radio map. This can be done with the Raspberry Pi units as their true coordinates are known in the map. In addition, the RSS values they exchanged between them during the experiment are also available. So, based on those Access Points the function can be built for the interpolation.

As aforementioned, the true distance between each Raspberry Pi is known. So thanks to the relationship between the RSS values given in equation (1) the distance that signals exchanged can be covered to be received.

$$
P_{\text {received }}=P_{\text {transmitted }}+20 \log (\lambda /(4 \pi d))
$$

where $d$ is the distance covered by the signal and $\lambda$ is the wavelength in this logarithmic path loss model.

But when we compare this distance $d$ to the true distances, a difference is observed. That's totally normal, and this is wanted because even in perfect conditions, signals, when travelling in the indoor environment, will undergo some issues like noise or spontaneous fluctuations. Knowing their difference for each time epoch, a correction for each Access Point can be computed. Taking the example illustrated in Fig. 8 with four Access Points AP2, AP4, AP5 and AP6 which communicate between each other and receive Wi-Fi signals from the other Raspberry Pi units and knowing their true distances, then from the theoretical RSS (RSS_t values which they should have obtained a RSS correction value $\mathrm{dR}$ can be computed in comparison with the measured RSS $m$ easured (RSS_m ).

As shows Fig. 8, the smartphone to be positioned is receiving signals from the Access Points and records their RSS values (i.e. the RSS_m). But due to noise and other influences, RSS $m$ will be different from the theoretical RSS (RSS $t$ ) that the smartphone should have received. Then if the distance with this RSS is directly computed, a wrong distance will obtained. But as for the Raspberry Pi units, the RSS values can be corrected as performing an interpolation. A simple way is given in equation (2):

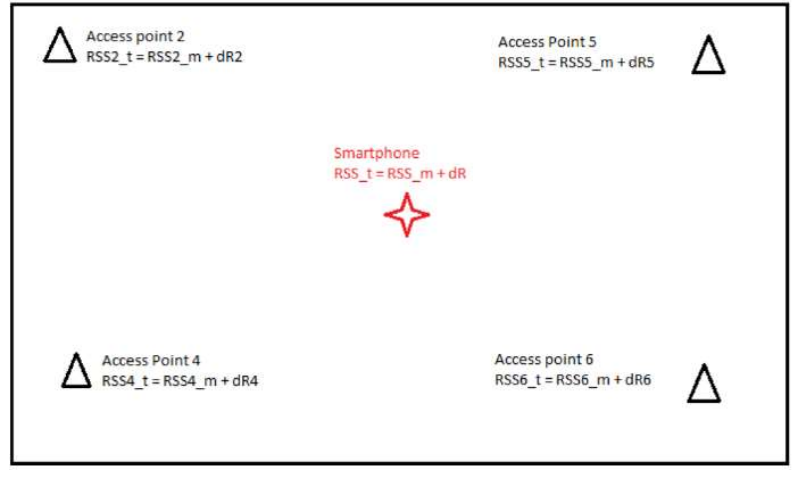

Fig. 8. DWi-Fi principle of operation.

$$
d R=w_{2} \times d R_{2}+w_{4} \times d R_{4}+w_{5} \times d R_{5}+w_{6} \times d R_{6}
$$

where $w$ are the weights affected at the correction from a certain Access Point (i.e., AP2, AP4, AP5 and AP6).

Then different ways could be taken into account to calculate such a correction:

- Equiprobability: all weights are the same,

- Path loss impact: adapt the weight taking into account the distance covered by the signal (an Access point nearer than the others will contribute more for the correction),

- Reliability: if an Access Point has a high variance, its impact in the final smartphone correction is diminished.

To start with, the equiprobability to compute the corrections between the Raspberry Pi's and for the smartphone was opted. For further information the detailed stepwise procedure to derive the correction parameters can be found [16].

\section{Conclusions and Outlook}

In this paper, key parameters and requirements as given for GNSS in the market report published in 2017 [1] are identified for indoor positioning using Wi-Fi and UWB. Field tests in an indoor market scenario were carried out and are reported here. The experimental area was covered with Raspberry Pi units serving as Wi-Fi Access Points and reference stations at the same time broadcasting and scanning the Wi-Fi signals of all available Raspberry Pi's and Wi-Fi routers. At the same location UWB transmitters, the anchors, were installed. Seven smartphones were employed to test the positioning solution.

In the analyses, the main parameters were the ID of the incoming Wi-Fi signal, its RSS and quality values, and the time where it was recorded. The signals exchanged between the Raspberry Pi units undergo several issues like not being with the same level of power according to the direction where they go. In addition, noise can interfere RSS measurements. However, corrections are derived from the known bias and the current RSS measurements thanks to Differential Wi-Fi. Work still to be done, is the interpolation of the RSS function $f(x, y)$ to determine the 
RSS value for each point in the radio map. This task could be done with Voronoï/Delaunay algorithms. It could be identified that especially in environments which are not suitably covered with Wi-Fi APs the use of Raspberry Pi units is economic.

A further task of future work is the integration of the UWB ranges into the overall positioning solution to increase the key parameters such as reliability and integrity.

With the solution proposed in this paper a step forward to GNSS-like positioning performance in indoor and combined transitional out-/indoor environments is achieved. Then for safety and liability critical applications, such as indoor navigation for the vision impaired and other mobility restricted individuals, emergency responders and asset tracking in buildings, a better performance is enabled.

The tests in QVM built the basis for an extensive measurement campaign conducted at the Ohio State University in October 2017 as part of the joint FIG Working Group 5.5 and IAG Sub-commission 4.1 on multi-sensor systems. A description of the experimental set-ups and characteristics of this campaign can be found in [8].

\section{References}

1. 2017 GNSS Market Report, https://www.gsa.europa.eu/2017-gnss-market-report (accessed October 2018).

2. B. Dewberry, A. Petroff, Precison Navigation with AD-HOC Autosurvey using UltraWideBand TwoWay Ranging Network, 12th IEEE Workshop on Positioning, Navigation and Communication WPNC'15, Dresden, Germany, March 11-12, 6 pgs. (2015).

3. S. Fortune, Voronoi Diagrams and Delaunay Triangulations, in: Handbook of Discrete and Computational Geometry, DOI:10.1201/ 9781420035315.ch23 (2004).

4. S. Goel, A Distributed Cooperative UAV Swarm Localization System: Development and Analysis, Proceedings of the 30th International Technical Meeting of the Satellite Division of the Institute of Navigation (ION GNSS+ 2017), Portland, Oregon, September 25-29, 2501-2518 (2017).

5. S. Goel, A. Kealy, B. Lohani, Development and Experimental Evaluation of a Low-Cost Cooperative UAV Localization Network Prototype, Journal of Sensor and Actuator Networks, 7(4), DOI: 10.3390/jsan7040042 (2018).

6. S. Goel, A. Kealy, G. Retscher, B. Lohani, Cooperative P2I Localization using UWB and Wi-Fi, Proceedings of the International Global Navigation Satellite Systems Association (IGNSS) Conference 2016, December 6-8, Sydney, Australia (2016).

7. T. Gonzales, Indoor Positioning Based on Differential Wi-Fi Measurements as well as GNSS
Integrity, Internship Report, RMIt University and The University of Melbourne, 21 pgs. (2018).

8. A. Kealy, G. Retscher, J. Gabela, Y. Li, S. Goel, C. K. Toth, A. Masiero, W. Błaszczak-Bąk, V. Gikas, H. Perakis, Z. Koppanyi, D. A. Grejner-Brzezinska, A Benchmarking Measurement Campaign in GNSSdenied/challenged Indoor/Outdoor and Transitional Environments, Proceedings of the FIG Working Week, April 22-26, Hanoi, Vietnam, 15 pgs. (2019) (submitted and under review).

9. H. Ledoux, C. Gold, An Efficient Natural Neighbour Interpolation Algorithm for Geoscientific Modelling, https://pdfs.semanticscholar.org/52ca/255573eded0e 4371fe2ced980b196636718d.pdf (accessed October 2018).

10. M. Lee, D. Han, Voronoi Tessellation Based Interpolation Method for Wi-Fi Radio Map Construction, IEEE Communications Letter, 16:3, 404-407 (2012).

11. R. Mautz, Indoor Positioning Technologies, Swiss Geodetic Commission, Geodetic-Geophysical Reports of Switzerland, 86, 134 pgs. (2012).

12. G. Retscher, Fusion of Location Fingerprinting and Trilateration Based on the Example of Wi-Fi Positioning. ISPRS Annals of the Photogrammetry, Remote Sensing and Spatial Information Sciences, Volume IV-2/W4, 2017, ISPRS Geospatial Week 2017, 18-22 September Wuhan, PR China, DOI 10.5194/isprs-annals-IV-2-W4-377-2017, pp. 377384 (2017).

13. G. Retscher, H. Hofer, A. Kealy, V. Gikas, F. Obex, Cooperative Localization in Indoor Environments Using Constrained Differential Wi-Fi and UWB Measurements, Proceedings of the ION GNSS+ Conference, Institute of Navigation: Portland, Oregon, USA (2017).

14. G. Retscher, A. Leb, Influence of the RSSI Scan Duration of Smartphones in Kinematic Wi-Fi Fingerprinting, Proceedings of the FIG Working Week, April 22-26, Hanoi, Vietnam, 15 pgs. (2019) (submitted and under review).

15. G. Retscher, Y. Li, S. Williams, A. Kealy, B. Moran, S. Goel, J. Gabela, Wi-Fi Positioning Using a Network Differential Approach for Real-time Calibration, Proceedings of the IGNSS 2018 Conference, February 7-9, 2018, Sydney, Australia, 15 pgs (2018).

16. G. Retscher, Y. Li, A. Kealy, H. Hofer, J. Gabela, S. Goel, O. Qureshi, E. Smith, L. Bao, Real-time Wi-Fi RSS Variation Correction Using a Network Differential Positioning Approach, Proceedings of the 9th International Conference Indoor Positioning and Indoor Navigation IPIN, September 24-27, Nantes, France, 4 pgs. (2018).

17. G. Retscher, T. Tatschl, Indoor Positioning with Differential Wi-Fi Lateration, Journal of Applied Geodesy, ISSN 1862-9016, DOI 10.1515/jag-20170011, 11:4, 249-269 (2017). 